\title{
The rate of decay of stable periodic solutions for Duffing equation with $L^{p}$-conditions
}

\author{
Shuqing Liang
}

\begin{abstract}
For a new class of $g(t, x)$, the existence, uniqueness and stability of $2 \pi$-periodic solution of Duffing equation $x^{\prime \prime}+c x^{\prime}+g(t, x)=h(t)$ are presented. Moreover, the unique $2 \pi$-periodic solution is (exponentially asymptotically stable) and its rate of exponential decay $c / 2$ is sharp. The new criterion characterizes $g_{x}^{\prime}(t, x)-c^{2} / 4$ with $L^{p}$-norms $(p \in[1, \infty])$, and the classical criterion employs the $L^{\infty}$-norm. The advantage is that we can deal with the case that $g_{x}^{\prime}(t, x)-c^{2} / 4$ is beyond the optimal bounds of the $L^{\infty}$-norm, because of the difference between the $L^{p}$-norm and the $L^{\infty}$-norm.
\end{abstract}

Mathematics Subject Classification. Primary 34C10; Secondary 34C10, $34 \mathrm{C} 25$.

Keywords. Duffing equation, Hill's equation, Periodic solutions, Stability, Decay rate.

\section{Introduction}

This paper deals with the existence, uniqueness, stability and the rate of decay of $2 \pi$-periodic solution of Duffing equation

$$
x^{\prime \prime}+c x^{\prime}+g(t, x)=h(t)
$$

for a new class of $g(t, x)$ characterized by $L^{p}$-norms, where $c>0$ is a constant, $g(t, x)$ is continuously differentiable and $2 \pi$-periodic in $t$, and $h(t)$ is continuous and $2 \pi$-periodic in $t$.

The existence and multiplicity of $T$-periodic solutions of (1.1) and more general equations have drawn a lot of attentions in the past three decades $[9,12,13,15,18-20,25,29,31,40]$. For more details, we refer to the survey of Mawhin [22]. However, the stability of periodic solutions is less extensively

The research is supported by NSFC No. 11501240, 11126043, SRFDP No. 20110061120003, and PSFC No. 2013M530970. This paper is a part of the author's dissertation [17]. 
studied $[3,4,27,33]$. Ortega $[26,28]$ study asymptotical stability of $T$-periodic solutions of (1.1) by topological degree. He investigate the connections between the stability and topological index of $T$-periodic solutions of the linear equation

$$
x^{\prime \prime}+c x^{\prime}+a(t) x=0,
$$

provided

$$
a(t)-\frac{c^{2}}{4}<\frac{\pi^{2}}{T^{2}}
$$

Lazer and McKenna [14] establish the stability of $T$-periodic solutions of (1.1) with linearization technique. Alonso and Ortega [1] prove the stability of $T$ periodic solutions of (1.1) and get some sharp bounds that guarantee global asymptotically stability. Njoku and Omari [24] present the existence and stability of $T$-periodic solutions of (1.1) with the method of upper and lower solutions. Recently, Chen and $\mathrm{Li}[5,6]$ prove that (1.1) has a unique and stable $T$-periodic solution $x_{0}(t)$, and every solution other than the unique $T$-periodic solution $x_{0}(t)$ exponentially decays to $x_{0}(t)$ at the sharp rate $c / 2$ (see $[5,6]$ and Remark 1.3), under the classical conditions

$$
\beta(t)+\frac{c^{2}}{4}<g_{x}^{\prime}(t, x)<\frac{\pi^{2}}{T^{2}}+\frac{c^{2}}{4}, \quad \text { and } \quad 0<\int_{0}^{T} \beta(t) d t
$$

or

$$
\frac{n^{2} \pi^{2}}{T^{2}}+\frac{c^{2}}{4}<g_{x}^{\prime}(t, x)<\frac{(n+1)^{2} \pi^{2}}{T^{2}}+\frac{c^{2}}{4}, \quad n=1,2, \ldots
$$

For convenience of notations, we set $G(t, x)=g_{x}^{\prime}(t, x)-c^{2} / 4$. According to (1.3) and (1.4), Floquet multipliers of the Hill's equation

$$
u^{\prime \prime}+G\left(t, x_{0}(t)\right) u=0
$$

are not real number, which is important for acquiring the rate of exponential decay of $x_{0}(t) c / 2$. In (1.4), $G(t, x)$ is bounded by $n^{2} \pi^{2} / T^{2}$ and $(n+1)^{2} \pi^{2} / T^{2}$ (points of resonance i.e. two constant functions of $t$ ). And in (1.3), the upper bound of $\pi^{2} / T^{2}$ is optimal in the sense of the $L^{\infty}$-norm, by the examples in $[5,6,28]$. The question arises whether we have some conclusions or not if $G(t, x)$ is beyond the optimal bounds of the $L^{\infty}$-norm. Similar ideas have been posed several times. For example, Llibre and Roberto [19], Torres and Zhang [30,32], Wang and $\mathrm{Li}[34]$ investigate the multiplicity and stability of $T$-periodic solutions of second order equation, with $L^{p}$-conditions for $g_{x}^{\prime}(t, x)$. Zhang and $\mathrm{Li}[36,38,39]$ develop some new estimates of the periodic and anti-periodic eigenvalues for the Hill's equation

$$
x^{\prime \prime}+a(t) x=0,
$$

with $L^{p}$-conditions $(p \in[1, \infty])$ for $a(t)=a(t+T)$. The author [16] get the exact multiplicities and stability of $2 \pi$-periodic solutions of $(1.1)$, with $L^{p}$ conditions for $g_{x}^{\prime}(t, x)$.

The purpose of this paper is to prove that (1.1) has a unique and (exponentially asymptotically stable) $2 \pi$-periodic solution, and its rate of exponential decay is $c / 2$ and is sharp. The criterion is that we illustrates $G(t, x)$ with $L^{p}$-norms $(p \in[1, \infty])$, and the advantage is that $G(t, x)$ can cross the bounds of the $L^{\infty}$-norm in (1.3) or (1.4), since the $L^{p}$-norm of a function is 
an integral but the $L^{\infty}$-norm of the function is the supremum of its range. The proof is based on Hartman-Grobman theorem, topological degree and the estimates for the periodic and anti-periodic eigenvalues of Hill's equation by $L^{p}$-norms $(p \in[1, \infty])$.

Some notations will be used throughout this paper.

$L^{p}(\mathbb{R} / 2 \pi \mathbb{Z}): 2 \pi$-periodic function $x \in L^{p}[0,2 \pi]$ with $\|x\|_{L^{p}[0,2 \pi]}, p \in[1, \infty]$.

$C^{k}(\mathbb{R} / 2 \pi \mathbb{Z}): 2 \pi$-periodic function $x \in C^{k}([0,2 \pi])$ with $\|x\|_{C^{k}}(k \geq 0)$. $\alpha(t) \succ \beta(t)$ : if $\alpha \geq \beta$ on $[0,2 \pi]$ and $\alpha>\beta$ on some subset of positive measure.

A $2 \pi$-periodic solution $x_{0}(t)$ of $(1.1)$ is said to be (locally exponentially asymptotical stable) if the following statement holds. There exist some constants $C>0$ and $\alpha>0$ such that any solution of (1.1) fulfilling $\left\|x(0)-x_{0}(0)\right\|+\left\|x^{\prime}(0)-x_{0}^{\prime}(0)\right\|=d$ sufficiently small, satisfies $\left\|x(t)-x_{0}(t)\right\|+$ $\left\|x^{\prime}(t)-x_{0}^{\prime}(t)\right\| \leq C d e^{-\alpha t}, \forall t \in(0,+\infty)$. The coefficient $\alpha$ in the exponent of this upper bound is called the rate of exponential decay of $2 \pi$-periodic solution $x_{0}(t)$.

The rate of exponential decay $c / 2$ is said to be sharp in the following sense. Consider the second-order differential equation

$$
x^{\prime \prime}+c x^{\prime}+k x=h(t),
$$

where $c>0$. If $k \neq 0$, then (1.5) has a unique $2 \pi$-periodic solution $x_{0}(t)$. [If $k \neq 0$ and $k \neq c^{2} / 4$, then each solution of (1.5) has the form $x(t)=$ $c_{1} e^{\rho_{1} t}+c_{2} e^{\rho_{2} t}+x_{0}(t)$, where $\rho_{1}, \rho_{2}$ are roots of $\rho^{2}+c \rho+k=0$.] If $k<0, x_{0}(t)$ is unstable. If $0<k<c^{2} / 4, x_{0}(t)$ is stable and its rate of decay is less than $c / 2$. [If $k=c^{2} / 4, x(t)=c_{1} e^{\rho t}+c_{2} t e^{\rho t}+x_{0}(t), \rho=-c / 2$, then $x_{0}(t)$ is stable and its rate of decay is less than $c / 2$.] If $c^{2} / 4<k^{2}$, then $\left|\rho_{1}\right|=\left|\rho_{2}\right|=-c / 2$, $x_{0}(t)$ is stable and its rate of decay is $c / 2$. Hence, if (1.5) has a unique and stable $2 \pi$-periodic solution, its rate of exponential decay $c / 2$ is sharp. We wish that the same conclusion for Eq. (1.1) holds.

In order to present the following class $\mathcal{Q}(p, n)$, we should recall Sobolev constants (see Zhang [37]). Let $p \in[1, \infty]$ and $I=\left[t_{0}, t_{1}\right]$ be a finite interval with the length denoted by $|I|$. Let $K(p,|I|)$ be the best Sobolev constant in the following inequality

$$
C\|u\|_{L^{p}(I)}^{2} \leq\left\|u^{\prime}\right\|_{L^{2}(I)}^{2} \quad \text { for all } u \in H_{0}^{1}(I) .
$$

Namely,

$$
K(p,|I|)=\inf _{u \in H_{0}^{1}(I) \backslash\{0\}}\left\|u^{\prime}\right\|_{L^{2}(I)}^{2} /\|u\|_{L^{p}(I)}^{2} .
$$

According to the Lemma 2.1 of Zhang [37], we know

$$
K(p,|I|)=K(p) /|I|^{1+2 / p},
$$

where

$$
K(p)= \begin{cases}\left(\frac{2(1+p / 2)^{1 / p} B(1 / p, 1 / 2)}{p(1+2 / p)^{1 / 2}}\right)^{2}, & 1 \leq p<+\infty, \\ 4, & p=+\infty\end{cases}
$$

and $B(\cdot, \cdot)$ is the Beta function of Euler. In particular, $K(2)=\pi^{2}, K(2,2 \pi)$ $=1 / 4$. Furthermore, if $p \in[1, \infty)$, the infimum in (1.6) can be attained by the 
function

$$
v_{p}(t)=\left\{\begin{array}{l}
E_{p}^{-1}\left(2\left(t-t_{0}\right) E_{p}(1) /|I|\right), t \in\left[t_{0},\left(t_{0}+t_{1}\right) / 2\right] \\
E_{p}^{-1}\left(2\left(t_{1}-t\right) E_{p}(1) /|I|\right), t \in\left[\left(t_{0}+t_{1}\right) / 2, t_{1}\right]
\end{array}\right.
$$

where $E_{p}:[0,1] \rightarrow \mathbb{R}$ is given by $E_{p}(t)=\int_{0}^{t} \frac{d x}{\left(1-x^{p}\right)^{1 / 2}}$. Then we acquire $\left\|v_{p}^{\prime}\right\|_{L^{2}(I)}^{2} /\left\|v_{p}\right\|_{L^{p}(I)}^{2}=K(p,|I|)$. If $|I|=1$ and $1 \leq p<\tilde{p} \leq \infty$, we get $K(p)=K(p,|I|), K(\tilde{p})=K(\tilde{p},|I|)$ and

$$
\left\|v_{p}\right\|_{L^{p}(I)}<|I|^{p \tilde{p} /(\tilde{p}-p)}\left\|v_{p}\right\|_{L^{\tilde{p}}(I)}=\left\|v_{p}\right\|_{L^{\tilde{p}}(I)},
$$

by the Hölder inequality. Thus,

$$
K(\tilde{p}) \leq\left\|v_{p}^{\prime}\right\|_{L^{2}(I)}^{2} /\left\|v_{p}\right\|_{L^{\tilde{p}}(I)}^{2}<\left\|v_{p}^{\prime}\right\|_{L^{2}(I)}^{2} /\left\|v_{p}\right\|_{L^{p}(I)}^{2}=K(p),
$$

and $K(p)$ is decreasing as a function of $p \in[1, \infty]$ (for more details, see Lemma 2.1 and Lemma 2.3 of Zhang [37]).

Let us present the following class $\mathcal{Q}(p, n)$ for the function $a(t), p \in[1, \infty]$ and $n \in[1, N(p)), n \in \mathbb{N}(3 \leq N(p)$ will be defined in the following). If $2 \leq n<N(p)$, we set

$$
\begin{aligned}
\mathcal{Q}(p, n) & =\left\{a \in L^{p}(\mathbb{R} / 2 \pi \mathbb{Z}): \frac{(n-1)^{2}}{4} \prec a \text { and }\|a\|_{p}<n^{2} K\left(2 p^{*}, 2 \pi\right)\right\}, p \in(1, \infty), \\
\mathcal{Q}(1, n) & =\left\{a \in L^{1}(\mathbb{R} / 2 \pi \mathbb{Z}): \frac{(n-1)^{2}}{4} \prec a \text { and }\|a\|_{1}<n^{2} K(\infty, 2 \pi)\right\}, \\
\mathcal{Q}(\infty, n) & =\left\{a \in L^{\infty}(\mathbb{R} / 2 \pi \mathbb{Z}): \frac{(n-1)^{2}}{4} \prec a \text { and } a \prec n^{2} K(2,2 \pi)=\frac{n^{2}}{4}\right\} .
\end{aligned}
$$

If $n=1$, we set

$$
\begin{aligned}
\mathcal{Q}(p, 1) & =\left\{a \in L^{p}(\mathbb{R} / 2 \pi \mathbb{Z}): 0<[a] \text { and }\left\|a_{+}\right\|_{p}<K\left(2 p^{*}, 2 \pi\right)\right\}, p \in(1, \infty), \\
\mathcal{Q}(1,1) & =\left\{a \in L^{1}(\mathbb{R} / 2 \pi \mathbb{Z}): 0<[a] \text { and }\left\|a_{+}\right\|_{1}<K(\infty, 2 \pi)\right\}, \\
\mathcal{Q}(\infty, 1) & =\left\{a \in L^{\infty}(\mathbb{R} / 2 \pi \mathbb{Z}): 0<[a] \text { and } a \prec K(2,2 \pi)=1 / 4\right\},
\end{aligned}
$$

where $[a]=(1 / 2 \pi) \int_{0}^{2 \pi} a(t) d t, a_{+}=\max \{a, 0\}, p^{*}=p /(p-1) \in[1, \infty]$. For $n=1$ and $p \in[1, \infty]$, we can easily check that the set $\mathcal{Q}(p, 1)$ is non empty. For $n \geq 2$ and $p \in[1, \infty], \mathcal{Q}(p, n)$ is not empty if and only if there exists function $a(t)$ satisfies

$$
(n-1)^{2} / 4 \prec a \quad \text { and } \quad\|a\|_{p}<n^{2} K\left(2 p^{*}, 2 \pi\right),
$$

which implies

$$
\left\|(n-1)^{2} / 4\right\|_{p}<\|a\|_{p}<n^{2} K\left(2 p^{*}, 2 \pi\right) .
$$

Note that $K(2)=\pi^{2}$, we obtain

$$
\left\|\frac{(n-1)^{2}}{4}\right\|_{p}=\frac{(n-1)^{2}}{4}(2 \pi)^{\frac{1}{p}}=(n-1)^{2} \frac{K(2)}{(2 \pi)^{2}}(2 \pi)^{\frac{1}{p}}=(n-1)^{2} \frac{K(2)}{(2 \pi)^{2-\frac{1}{p}}} .
$$

It follows form $(1.7)$ and $1 / p+1 / p^{*}=1$ that

$$
n^{2} K\left(2 p^{*}, 2 \pi\right)=n^{2} \frac{K\left(2 p^{*}\right)}{(2 \pi)^{1+\frac{2}{2 p^{*}}}}=n^{2} \frac{K\left(2 p^{*}\right)}{(2 \pi)^{1+1-\frac{1}{p}}}=n^{2} \frac{K\left(2 p^{*}\right)}{(2 \pi)^{2-\frac{1}{p}}} .
$$


Therefore, (1.9) is equivalent to

$$
1-1 / n=(n-1) / n<\sqrt{K\left(2 p^{*}\right) / K(2)} .
$$

If $p=\infty$, the right hand side of (1.10) is $1,(1.10)$ is satisfied for all $n \in \mathbb{Z}^{+}$. If $p \in[1, \infty)$, the right hand side of $(1.10)$ is strictly less than 1 , since $K(p)$ is decreasing function of $p \in[1, \infty]$. Apparently, we can write (1.10) as

$$
n<1 /\left(1-\sqrt{K\left(2 p^{*}\right) / K(2)}\right):=k_{0}(p) .
$$

As $K(p)$ is decreasing, we know $K(\infty) \leq K\left(2 p^{*}\right)$ for $p \in[1, \infty]$, and

$$
k_{0}(p) \geq k_{0}(1)=1 /(1-\sqrt{K(\infty) / K(2)})=1 /\left(1-\sqrt{4 / \pi^{2}}\right)>2 .
$$

According to Zhang [37], one can define the number

$$
N(p)= \begin{cases}\infty & \text { if } p=\infty \\ {\left[k_{0}(p)\right]} & \text { if } p \in[1, \infty) \text { and } k_{0}(p) \text { is an integer } \\ {\left[k_{0}(p)\right]+1} & \text { if } p \in[1, \infty) \text { and } k_{0}(p) \text { is not an integer }\end{cases}
$$

where $\left[k_{0}(p)\right]$ stands for the integer part of $k_{0}(p)$. The definition of $N(p)$ and (1.11) lead to $N(p) \geq 3$. Therefore, for $2 \leq n<N(p)$ and $p \in[1, \infty], \mathcal{Q}(p, n)$ is also not empty, on account of the definition of $N(p),(1.10)$ and (1.9).

Nowadays, we are in a position to present the main result of this paper. Let us recall $G(t, x)=g_{x}^{\prime}(t, x)-c^{2} / 4$, which is a function of $t$ and $x$.

Theorem 1.1. Assume that $g(t, x) \in C^{1}(\mathbb{R} / 2 \pi \mathbb{Z} \times \mathbb{R})$ and $l(t) \leq G(t, x) \leq u(t)$, $\forall x \in \mathbb{R}$, where $2 \pi$-periodic continuous functions $l(t), u(t) \in \mathcal{Q}(p, n), p \in$ $[1, \infty], n \in[1, N(p)), n \in \mathbb{N}$. Then Eq. (1.1) has a unique and (exponentially asymptotically stable) $2 \pi$-periodic solution $x_{0}(t)$. And the rate of exponential decay of $x_{0}(t)$ is $c / 2$ and is sharp.

Remark 1.2. To analyze the rate of exponential decay of $x_{0}(t)$, Chen and Li $[5,6]$ suppose (1.3) and (1.4). They are equivalent to the conditions that there exist functions $l(t), u(t) \in \mathcal{Q}(\infty, 1)$ or $\mathcal{Q}(\infty, n)$ such that $l(t) \leq G(t, x) \leq u(t)$, respectively, if $T=2 \pi$. To obtain the similar result, Theorem 1.1 assume that there exist $l(t), u(t) \in \mathcal{Q}(p, 1)$ or $\mathcal{Q}(p, n)$ such that $l(t) \leq G(t, x) \leq u(t)$. For the case of $G(t, x)<0$ (see (1.2)), we refer to Ortega [28], where he has investigated the exact multiplicity and stability of $T$-periodic solutions of (1.1).

Remark 1.3. Assuming (1.3) and $T=2 \pi$, the rate of exponential decay of $2 \pi$ periodic solution of $(1.1)$ is $c / 2$ (see also Chen and $\mathrm{Li}[5,6]$ ). However, when $G(t, x)$ is beyond $\pi^{2} /(2 \pi)^{2}=1 / 4$, the rate of decay of $2 \pi$-periodic solution of (1.1) could be less than $c / 2$ (see the examples of $[5,6,28]$ ). Therefore, if (1.1) has a unique and stable $2 \pi$-periodic solution, then the rate of exponential decay $c / 2$ is sharp. Meanwhile, one could state that (1.3) is optimal in the sense of the $L^{\infty}$-norm.

Remark 1.4. The following Example 1.5 presents a class of $G(t, x)$, where $G(t, x)$ is beyond the upper bound $n^{2} / 4$ of the criterion of $L^{\infty}$-norm. Hence, we can not find functions $l_{\infty}(t), u_{\infty}(t) \in \mathcal{Q}(\infty, n)$ such that $l_{\infty}(t) \leq G(t, x) \leq$ $u_{\infty}(t), \forall x \in \mathbb{R}$, and the classical criterion could not be applied. However, there 
exist $l_{p}(t), u_{p}(t) \in \mathcal{Q}(p, n)$ such that $l_{p}(t) \leq G(t, x) \leq u_{p}(t), \forall x \in \mathbb{R}$, hence Theorem 1.1 can be applied.

Example 1.5. For $n=1$, it is easy to check that $\mathcal{Q}(p, n)$ is not empty. For $n \in[2, N(p)), \mathcal{Q}(p, n)$ is not empty if and only if there exists function $a(t)$ that satisfies

$$
(n-1)^{2} / 4 \prec a \quad \text { and } \quad\|a\|_{p}<n^{2} K\left(2 p^{*}, 2 \pi\right)
$$

which implies

$$
(n-1)^{2}(2 \pi)^{1 / p} / 4=\left\|(n-1)^{2} / 4\right\|_{p}<\|a\|_{p}<n^{2} K\left(2 p^{*}, 2 \pi\right) .
$$

Let $g(t, x)=x\left(a(t) / 8+\left((n-1)^{2}+c^{2}\right) / 4\right)$, and $2 \pi$-periodic, positive and continuous function $a(t) \in L^{p}(\mathbb{R} / 2 \pi \mathbb{Z})$ satisfies $0<\min _{[0,2 \pi]} a(t)<$ $1 / 16<2\left(n^{2}-(n-1)^{2}\right)<\max _{[0,2 \pi]} a(t)=\|a\|_{\infty}$, and $\|a\|_{p}<n^{2} K\left(2 p^{*}, 2 \pi\right)-$ $(n-1)^{2}(2 \pi)^{\frac{1}{p}} / 4, n=1,2, \ldots$ Then, we have

$$
G(t, x)=g_{x}^{\prime}(t, x)-c^{2} / 4=\frac{a(t)}{8}+\frac{(n-1)^{2}+c^{2}}{4}-\frac{c^{2}}{4}=\frac{a(t)}{8}+\frac{(n-1)^{2}}{4} .
$$

Let $l_{\infty}(t) \equiv \min _{[0,2 \pi]} a(t) / 16+(n-1)^{2} / 4$, then $G(t, x)>l_{\infty}(t)$. Let $u(t) \equiv n^{2} / 4$, then there exists $s_{0} \in[0,2 \pi]$ and $x \in \mathbb{R}$ such that $G\left(s_{0}, x\right)<n^{2} / 4=u(t)$. And there exists $s_{1} \in[0,2 \pi]$ and $x \in \mathbb{R}$ such that $G\left(s_{1}, x\right)>n^{2} / 4=u(t)$, since

$$
\begin{aligned}
\|G(t, x)\|_{\infty} & =\left\|\frac{a(t)}{8}+\frac{(n-1)^{2}}{4}\right\|_{\infty}=\left\|\frac{a(t)}{8}\right\|_{\infty}+\frac{(n-1)^{2}}{4} \\
& >\frac{n^{2}-(n-1)^{2}}{4}+\frac{(n-1)^{2}}{4}=\frac{n^{2}}{4}=u(t) .
\end{aligned}
$$

Hence, $G(t, x)$ crosses $u(t) \equiv n^{2} / 4$, and we can not find functions $l_{\infty}(t), u_{\infty}(t)$ $\in \mathcal{Q}(\infty, n)$ such that $l_{\infty}(t) \leq G(t, x) \leq u_{\infty}(t)$.

On the other hand, let $l_{p}(t)=l_{\infty}(t)$, and $u_{p}(t)=a(t) / 2+(n-1)^{2} / 4$. We know $(n-1)^{2} / 4<l_{p}(t) \leq G(t, x) \leq u_{p}(t)$ and

$$
\begin{aligned}
\|G(t, x)\|_{p} & =\left\|\frac{a(t)}{8}+\frac{(n-1)^{2}}{4}\right\|_{p}<\left\|\frac{a(t)}{2}+\frac{(n-1)^{2}}{4}\right\|_{p}=\left\|u_{p}(t)\right\|_{p} \\
& <\|a(t)\|_{p}+\left\|\frac{(n-1)^{2}}{4}\right\|_{p}<n^{2} K\left(2 p^{*}, 2 \pi\right) .
\end{aligned}
$$

Hence, we know $l_{p}(t), u_{p}(t) \in \mathcal{Q}(p, n)$. Consequently, there exist $l_{p}(t), u_{p}(t) \in$ $\mathcal{Q}(p, n)$ such that $l_{p}(t) \leq G(t, x) \leq u_{p}(t), \forall x \in \mathbb{R}$.

Example 1.6. We can consider $g(t, x)=a(t)(x-\arctan x / 4)+x\left((n-1)^{2}+c^{2}\right) / 4$ by similar estimates, and let us omit the details.

This paper is organized as follows. In Sect. 2, some fundamental results about topological degree, the connections between the rotation numbers and the periodic and anti-periodic eigenvalues of the Hill's equation are presented. In Sect. 3, the proof of the main theorem is established. 


\section{Preliminaries}

\subsection{Continuation theorem for periodic problems}

Consider the periodic boundary value problem

$$
\begin{aligned}
x^{\prime} & =f(t, x), \\
x(0) & =x(2 \pi),
\end{aligned}
$$

where $f: \mathbb{R} / 2 \pi \mathbb{Z} \times \mathbb{R}^{2} \rightarrow \mathbb{R}^{2}$ is a continuously differentiable function and $2 \pi$-periodic in $t$. The topological degree can be computed by the homotopy

$$
\hat{f}(t, x, \lambda)=\lambda f(t, x)+(1-\lambda) \tilde{f}(x), \quad \lambda \in[0,1],
$$

where $\tilde{f}(x)$ is a continuous function. The following continuation theorem of Mawhin is needed for the problem of (2.1)-(2.2).

Lemma 2.1. [23] Let $\Omega \subset C[0,2 \pi]$ be open bounded set such that the following conditions are satisfied.

(i) There is no $x \in \partial \Omega$ such that $x^{\prime}=\hat{f}(t, x, \lambda), \forall \lambda \in[0,1)$;

(ii) $\operatorname{deg}\left(\tilde{f}, \Omega \cap \mathbb{R}^{2}, 0\right) \neq 0$.

Then (2.1)-(2.2) has at least one solution $x \in \Omega$.

Suppose the initial-value solution $x\left(t, x_{0}\right)$ of $(2.1)$ for $x(0)=x_{0} \in \mathbb{R}^{2}$ is defined for all $t \in \mathbb{R}$ and we have the Poincaré mapping $P: x_{0} \rightarrow x\left(2 \pi, x_{0}\right)$. ]]Then $x\left(t, x_{0}\right)$ is a $2 \pi$-periodic solution of $(2.1)-(2.2)$ if and only if $x_{0}$ is a fixed point of $P$. Moreover, if $x\left(t, x_{0}\right)$ is an isolated $2 \pi$-periodic solution of (2.1), then $x_{0}$ is an isolated fixed point of $P$.

A $2 \pi$-periodic solution $x$ of $(2.1)$ is nondegenerate, if the linearized equation

$$
y^{\prime}=f_{x}(t, x(t)) y
$$

does not admit any nontrivial $2 \pi$-periodic solutions.

Let $M(t)$ be the fundamental matrix solution of $(2.3)$ with $M(0)=I$. Then $M(2 \pi)$ is monodromy matrix of (2.3), and two eigenvalues $\mu_{1}$ and $\mu_{2}$ of it are the Floquet multipliers. If $\left|\mu_{i}\right|<1(i=1$ and 2$)$, then the $2 \pi$-periodic solution $x$ of (2.1) is asymptotically stable.

\subsection{The rotation number of Hill's equation}

Consider the Hill's equation (we refer to [21,38])

$$
x^{\prime \prime}+a(t) x=0,
$$

where $a(t) \in L^{1}(\mathbb{R} / 2 \pi \mathbb{Z})$. Let $M(t)$ is the fundamental matrix solution of (2.4) with $M(0)=I$. It follows from $\operatorname{det} M(2 \pi)=1$ that the eigenvalues $\mu_{1}, \mu_{2}$ of $M(2 \pi)$ verify $\mu_{1} \mu_{2}=1$. Equation (2.4) is called hyperbolic, elliptic or parabolic if $\left|\mu_{i}\right| \neq 1, \mu_{i} \in \mathbb{S}^{1} \backslash\{ \pm 1\}$ or $\mu_{1}=\mu_{2}= \pm 1$, respectively. Equation (2.4) is called nondegenerate if $\mu_{i} \neq 1(i=1$ and 2$)$, which implies that (2.4) has no nontrivial $2 \pi$-periodic solutions. 
Under the transformation $\left(x, x^{\prime}\right)=(r \cos \theta,-r \sin \theta)$, we know from (2.4) that argument $\theta=\theta(t)$ satisfies

$$
\theta^{\prime}=\cos ^{2} \theta+a(t) \sin ^{2} \theta .
$$

The rotation number of (2.4) is defined by

$$
\rho(a)=\lim _{t \rightarrow \infty} \theta(t) / t,
$$

which is independent of choice of solutions $\theta(t)$. Some properties of the rotation number of (2.4) are collected in the following lemma.

Lemma 2.2. $[10,38]$ Let $a \in L^{p}(\mathbb{R} / 2 \pi \mathbb{Z})$ for some $p \in[1, \infty]$. Then one has

(i) $0 \leq \rho=\rho(a) \leq \sqrt{\left\|a_{+}\right\|_{p}} /\left(2 \sqrt{K\left(2 p^{*}, 2 \pi\right)}\right)$;

(ii) If $a_{1}(t) \prec a_{2}(t)$, then $\rho\left(a_{1}\right)<\rho\left(a_{2}\right)$;

(iii) Equation (2.4) is elliptic if and only if $\rho \in((n-1) / 2, n / 2)$ for some $n \in \mathbb{N}$. And the Floquet multipliers of $(2.4)$ are $\mu_{1,2}=e^{ \pm i 2 \pi \rho}$;

(iv) Equation (2.4) is non-degenerate if $\rho \in(n-1, n)$ for some $n \in \mathbb{N}$.

\subsection{The periodic and anti-periodic eigenvalues of Hill's equation}

We recall the periodic and anti-periodic eigenvalues of Hill's equation (see $[2,10,11,38])$

$$
x^{\prime \prime}+(\lambda+a(t)) x=0
$$

with respect to the $2 \pi$-periodic boundary condition

$$
x(0)=x(2 \pi), \quad x^{\prime}(0)=x^{\prime}(2 \pi)
$$

or the $2 \pi$-antiperiodic boundary condition

$$
x(0)=-x(2 \pi), \quad x^{\prime}(0)=-x^{\prime}(2 \pi) .
$$

There exist sequences (see $[21,37]$ )

$$
-\infty<\bar{\lambda}_{0}(a)<\underline{\lambda}_{1}(a) \leq \bar{\lambda}_{1}(a)<\underline{\lambda}_{2}(a) \leq \bar{\lambda}_{2}(a)<\cdots<\underline{\lambda}_{k}(a) \leq \bar{\lambda}_{k}(a)<\cdots
$$

such that

(i) $\lambda$ is an eigenvalue of (2.5)-(2.6) if and only if $\lambda=\underline{\lambda}_{k}(a)$ or $\lambda=\bar{\lambda}_{k}(a)$ for $k=0,2,4, \ldots$

(ii) $\lambda$ is an eigenvalue of (2.5)-(2.7) if and only if $\lambda=\underline{\lambda}_{k}(a)$ or $\lambda=\bar{\lambda}_{k}(a)$ for $k=1,3,5, \ldots$.

(iii) Equation (2.5) is bounded if $\lambda$ is in the intervals

$$
\left(\bar{\lambda}_{0}(a), \underline{\lambda}_{1}(a)\right),\left(\bar{\lambda}_{1}(a), \underline{\lambda}_{2}(a)\right), \ldots,\left(\bar{\lambda}_{k}(a), \underline{\lambda}_{k+1}(a)\right), \ldots
$$

(iv) Equation (2.5) is unbounded if $\lambda$ is in the intervals

$$
\left(-\infty, \bar{\lambda}_{0}(a)\right),\left(\underline{\lambda}_{1}(a), \bar{\lambda}_{1}(a)\right), \ldots,\left(\underline{\lambda}_{k}(a), \bar{\lambda}_{k}(a)\right), \ldots
$$

According to the explanation for the class $\mathcal{Q}(p, n)$ in Introduction, the following lemma holds.

Lemma 2.3. Assume $p \in[1, \infty]$ and $n \in[1, N(p)), n \in \mathbb{N}$, then $\mathcal{Q}(p, n)$ is non-empty and convex set of $L^{p}(\mathbb{R} / 2 \pi \mathbb{Z})$.

The following lemma discusses the elliptic property and nondegeneracy of the Hill's equation (2.4). 
Lemma 2.4. Suppose that $a(t) \in \mathcal{Q}(p, n)$ for some $p \in[1, \infty], n \in$ $[1, N(p)), n \in \mathbb{N}$. Then (2.4) is elliptic and also non-degenerate.

Proof. Firstly, we consider the case of $n \in[2, N(p))$. It follows from $\frac{(n-1)^{2}}{4} \prec a$ and (ii) of Lemma 2.2 that $(n-1) / 2<\rho(a)$. Meanwhile, it follows from $\|a\|_{p}<n^{2} K\left(2 p^{*}, 2 \pi\right)$ and (i) of Lemma 2.2 that $\rho(a)<n / 2$. According to (iii) of Lemma 2.2, Eq. (2.4) is elliptic, since $\rho(a) \in((n-1) / 2, n / 2)$ for some $n \in \mathbb{N}$. Thus the conclusion holds.

Secondly, for the case of $n=1$, one has trivial upper bound for the smallest $2 \pi$-periodic eigenvalue $\bar{\lambda}_{0}(a) \leq-[a]<0$. Then, $\rho(a)>0$, by the definition of $2 \pi$-periodic eigenvalues of (2.4) and (ii) of Lemma 2.2. Meanwhile, it follows from $\left\|a_{+}\right\|_{p}<K\left(2 p^{*}, 2 \pi\right)$ and (i) of Lemma 2.2 that $\rho(a)<1 / 2$. Hence, one has $\rho(a) \in(0,1 / 2)$ and (2.4) is elliptic for the case of $n=1$. Therefore, the conclusions hold for the whole class of $\mathcal{Q}(p, n), p \in[1, \infty], n \in$ $[1, N(p)), n \in \mathbb{N}$.

\subsection{Homogeneous periodic equation}

Let us consider the homogeneous periodic equation

$$
L_{a} x:=x^{\prime \prime}+c x^{\prime}+a(t) x=0 .
$$

By the change of variables $u=e^{\frac{c t}{2}} x$, we transform (2.8) to the Hill's equation

$$
u^{\prime \prime}+\left(a(t)-\frac{c^{2}}{4}\right) u=0 .
$$

Lemma 2.5. Assume that $a(t)-c^{2} / 4 \in \mathcal{Q}(p, n)$ for some $p \in[1, \infty], n \in$ $[1, N(p)), n \in \mathbb{N}$. Then one has

(i) Equation (2.8) does not admit real Floquet multipliers and has no nontrivial $2 \pi$-periodic solutions.

(ii) The module of the Floquet multipliers of (2.8) are less than one. And the decay of exponential rate of any nontrivial solutions of (2.8) is c/2.

Proof. Suppose (2.8) has Floquet multiplier $\mu$, then (2.9) has Floquet multiplier $\mu e^{\pi c}$. It follows from elliptic property of the Hill's equation and Lemma 2.4 that (2.9) does not admit real Floquet multipliers. Thus (2.8) does not admit real multipliers, and has no nontrivial $2 \pi$-periodic solutions. Hence, (i) is clear.

Now, we prove (ii). We consider the planar system associate with (2.8)

$$
S^{\prime}(t)=A(t) S(t)
$$

where $S(t)=(x(t), y(t))^{T}$ and

$$
A(t)=\left(\begin{array}{cc}
0 & 1 \\
-a(t) & -c
\end{array}\right) .
$$

Let $M(t)$ be a fundamental matrix solution of (2.10) with $M(0)=I$. By the Floquet theory (see [21]), $M(t)$ has the form

$$
M(t)=P(t) e^{B t},
$$


where $P(t)=P(t+2 \pi)$ is $2 \times 2$ matrices, and $B$ is a $2 \times 2$ constant matrix. Let $\mu_{1}=e^{2 \pi \eta_{1}}, \mu_{2}=e^{2 \pi \eta_{2}}$ be the Floquet multipliers, $\eta_{1}$ and $\eta_{2}$ be the Floquet exponents associated with $\mu_{1}$ and $\mu_{2}$. It follows from (i) of this lemma that $\mu_{1}$ and $\mu_{2}$ are a pair of conjugate complex numbers and $\left|\mu_{1}\right|=\left|\mu_{2}\right|$. And the two eigenvectors of the matrix $e^{2 \pi B}$ are linearly independent. Applying the Jacobi-Liouville formula, we have

$$
e^{2 \pi\left(\eta_{1}+\eta_{2}\right)}=\left|\mu_{1}\right|^{2}=\mu_{1} \mu_{2}=e^{-\int_{0}^{2 \pi} c d t}=e^{-2 \pi c},
$$

and

$$
\operatorname{Re} \eta_{1}=\operatorname{Re} \eta_{2}=\frac{1}{2} \operatorname{Re}\left(\eta_{1}+\eta_{2}\right)=\frac{1}{4 \pi} \ln \left(\mu_{1} \mu_{2}\right)=-\frac{c}{2} .
$$

And (2.8) has linearly independent solutions $x_{i}(t)=p_{i}(t) e^{\eta_{i} t}, p_{i}(t)=p_{i}(t+2 \pi)$ $(i=1,2)$. Since every solution of (2.8) is a linear combination of $x_{1}(t)$ and $x_{2}(t)$, we know that every nonzero solution of (2.8) decays at the rate of exponential decay $c / 2$.

\section{The proof of main result}

The proof of Theorem 1.1 is divided in two steps. Firstly, we prove the existence, uniqueness and stability of $2 \pi$-periodic solution of (1.1). Define $F: C^{2}(\mathbb{R} / 2 \pi \mathbb{Z}) \rightarrow C(\mathbb{R} / 2 \pi \mathbb{Z})$ by

$$
F(x(t))=x^{\prime \prime}+c x^{\prime}+g(t, x(t)),
$$

and recall $G(t, x)=g_{x}^{\prime}(t, x)-c^{2} / 4$.

Lemma 3.1. Under the assumptions of Theorem 1.1, Eq. (1.1) has a unique and stable $2 \pi$-periodic solution $x_{0}(t)$.

Proof. Existence Without loss of generality, we may assume that $g(t, 0)=0$, for otherwise we minus both sides of $(1.1)$ by $g(t, 0)$. Consider the parameterized equation

$$
F_{\lambda}:=x^{\prime \prime}+c x^{\prime}+\lambda g(t, x)+(1-\lambda) b(t) x=\lambda h(t),
$$

where $b(t)-c^{2} / 4 \in \mathcal{Q}(p, n)$, for some $p \in[1, \infty], n \in[1, N(p))$.

We claim that $2 \pi$-periodic solutions of (3.1) are bounded in $L^{\infty}(\mathbb{R} / 2 \pi \mathbb{Z})$ and independent of $\lambda$. Assume by contradiction that there would exist sequences $\left\{\lambda_{n}, x_{n}\right\}$ of $2 \pi$-periodic solutions of (3.1) with $\left\|x_{n}\right\|_{\infty} \rightarrow+\infty$ and $\lambda_{n} \in[0,1]$. Denote by $z_{n}$ the quotient $z_{n}=x_{n} /\left\|x_{n}\right\|_{\infty}$ and $\left\|z_{n}\right\|_{\infty}=1$. Dividing (3.1) by $\left\|x_{n}\right\|_{\infty}$, one has

$$
z_{n}^{\prime \prime}+c z_{n}^{\prime}+\lambda_{n} \frac{g\left(t, x_{n}\right)}{\left\|x_{n}\right\|_{\infty}}+\left(1-\lambda_{n}\right) b z_{n}=\lambda_{n} \frac{h}{\left\|x_{n}\right\|_{\infty}} .
$$

Since there exist continuous $2 \pi$-periodic functions $l(t), u(t) \in \mathcal{Q}(p, n)$, such that $l(t)<G(t, x)=g_{x}^{\prime}(t, x)-c^{2} / 4<u(t), \forall x \in \mathbb{R}$, one has

$$
\left|\frac{g(t, x)}{x}\right|=\left|\frac{g(t, x)-g(t, 0)}{x-0}\right|=\left|g_{x}^{\prime}(t, \xi)\right|<|l(t)|+|u(t)|+\frac{c^{2}}{4}<C_{1} .
$$


It implies that the $L^{\infty}$-norm of $\left[\lambda_{n} g\left(t, x_{n}\right)+\left(1-\lambda_{n}\right) b(t) x_{n}\right] /\left\|x_{n}\right\|_{\infty}$ is bounded. Thus there exist subsequences such that $g\left(t, x_{n}\right) / x_{n} \stackrel{*}{\rightarrow} \tilde{b}(t)$ in $L^{\infty}(\mathbb{R} / 2 \pi \mathbb{Z})$ and $\lambda_{n} \rightarrow \lambda$, and $\tilde{b}(t) \in \mathcal{Q}(p, n)$. (We refer to Theorem 5.1 of [8] or Theorem 5.3.4 of [35]. If Banach space is separable, then the bounded set of its dual space is weak* sequentially compact. Since Banach space $L^{1}(\mathbb{R} / 2 \pi \mathbb{Z})$ is separable, and $L^{\infty}(\mathbb{R} / 2 \pi \mathbb{Z})$ is its dual space, we know that the bounded set $g\left(t, x_{n}\right) / x_{n}$ is weak* sequentially compact).

Since $z_{n}(t)$ is a $2 \pi$-periodic solution, there exists $t_{0}$ such that $z_{n}^{\prime}\left(t_{0}\right)=0$. Integrating (3.2) from $t_{0}$ to $t$ and using inequality (3.3), we have $\left\|z_{n}^{\prime}\right\|_{\infty}<M$. Using the Eq. (3.2) again, we know $\left\|z_{n}^{\prime \prime}\right\|_{\infty}<M$. It follows from Arzela-Ascoli theorem that there exist uniformly convergent subsequences on $[0,2 \pi]$ for $\left\{z_{n}\right\}$ and $\left\{z_{n}^{\prime}\right\}$, by taking a subsequence of necessary, such that

$$
\lim _{n \rightarrow \infty} z_{n}=z, \quad \lim _{n \rightarrow \infty} z_{n}^{\prime}=z^{\prime} .
$$

Multiplying both sides of $(3.2)$ by $\varphi(t) \in C^{2}(\mathbb{R} / 2 \pi \mathbb{Z})$ and integrating by parts, one has

$$
\int_{0}^{2 \pi} \frac{\left[\varphi^{\prime \prime}-c \varphi^{\prime}+\left(\lambda_{n} g\left(t, x_{n}\right) / x_{n}+\left(1-\lambda_{n}\right) b\right) \varphi\right] x_{n}}{\left\|x_{n}\right\|_{\infty}} d t=\lambda_{n} \int_{0}^{2 \pi} \frac{\varphi h}{\left\|x_{n}\right\|_{\infty}} d t .
$$

Based on the above analysis, one can take the limit in (3.4) and obtain

$$
\int_{0}^{2 \pi}\left[\varphi^{\prime \prime}-c \varphi^{\prime}+\hat{b}(t) \varphi\right] z d t=0
$$

where $\hat{b}(t)=\lambda \tilde{b}(t)+(1-\lambda) b(t)$. Obviously, $\hat{b}(t)-c^{2} / 4$ is a member of $\mathcal{Q}(p, n), p \in[1, \infty], n \in[1, N(p))$, and satisfies the assumptions of Lemma 2.5. It follows from Lemma 2.5 that the $2 \pi$-periodic solution $z(t) \equiv 0$, which contradicts the fact that $\|z(t)\|_{\infty}=1$. Hence, $2 \pi$-periodic solutions of $(3.1)$ are bounded in $L^{\infty}(\mathbb{R} / 2 \pi \mathbb{Z})$ and independent of $\lambda$.

Next, it follows from the homotopic invariance property that

$$
\operatorname{deg}\left(F_{0}, B_{R}, 0\right)=\operatorname{deg}\left(F_{1}, B_{R}, 0\right)=1 .
$$

According to Lemma 2.1, (1.1) has a $2 \pi$-periodic solution. This completes the proof of existence of $2 \pi$-periodic solutions.

Uniqueness Let $x_{1}(t)$ and $x_{2}(t)$ be two $2 \pi$-periodic solutions of (1.1), and $v(t)=x_{1}(t)-x_{2}(t)$. Then $v(t)$ satisfies

$$
v^{\prime \prime}+c v^{\prime}+e(t) v=0,
$$

where $e(t)=\int_{0}^{1} g_{x}^{\prime}\left(t, x_{2}(t)+s v(t)\right) d s$. It follows from Lemma 2.5 that $v(t) \equiv 0$, hence $x_{1}(t) \equiv x_{2}(t)$.

Stability Let $x_{0}(t)$ be the unique $2 \pi$-periodic solution. Consider the linearized equation

$$
w^{\prime \prime}+c w^{\prime}+g_{x}^{\prime}\left(t, x_{0}(t)\right) w=0,
$$

where $g_{x}^{\prime}\left(t, x_{0}(t)\right)$ is a function of $t$. However, the assumption of $l(t) \leq G(t, x) \leq$ $u(t), \forall x \in \mathbb{R}$ implies that $l(t) \leq g_{x}^{\prime}\left(t, x_{0}(t)\right)-c^{2} / 4=G\left(t, x_{0}(t)\right) \leq u(t)$, then $G\left(t, x_{0}(t)\right) \in \mathcal{Q}(p, n)$ and the assumption of Lemma 2.5 is verified. Then, Lemma 2.5 implies the stability results of the linearized equation. 
Secondly, we show that every solution of (1.1) locally decays to the unique $2 \pi$-periodic solution $x_{0}(t)$ at the rate of exponential decay $c / 2$. The following $C^{1}$ version of the Hartman-Grobman theorem is needed.

A transformation $f: \mathbb{R}^{2} \rightarrow \mathbb{R}^{2}$ is called contracting mapping, if there is a $\lambda \in[0,1)$ such that $|f(x)-f(y)| \leq \lambda|x-y|, \forall x, y \in \mathbb{R}^{2}$. The constant $\lambda$ is called the contraction constant for $f$ on $\mathbb{R}^{2}$.

Lemma 3.2. [7] Let $f: U \subset \mathbb{R}^{2} \rightarrow \mathbb{R}^{2}$ be a $C^{1}$ function with $f(0)=0$ and $f_{x}^{\prime}(0): \mathbb{R}^{2} \rightarrow \mathbb{R}^{2}$ a contracting mapping. Then $f$ is $C^{1}$-conjugate equivalent to $f_{x}^{\prime}(0)$.

Rate of decay We consider the planar system associated with Duffing equation $(1.1)$

$$
\left\{\begin{array}{l}
x^{\prime}=c x-y \\
y^{\prime}=h(t)-g(t, x) .
\end{array}\right.
$$

Assume $S(t)=(x(t), y(t))$ is the initial-value solution of (3.5) with $S(0)=$ $X \in \mathbb{R}^{2}$, we have the Poincaré mapping $P: X \rightarrow S(2 \pi, X)$. It follows from Lemma 3.1 that $(3.5)$ has a unique $2 \pi$-periodic solution $X_{0}(t)=\left(x_{0}(t), y_{0}(t)\right)$. Then $X_{0}=\left(x_{0}(0), y_{0}(0)\right)=\left(x_{0}, y_{0}\right)$ is the unique fixed point of the Poincaré mapping $P$. Let $M(t)$ be the fundamental matrix solution of the linearization of $(3.5)$

$$
\tilde{S}^{\prime}=B(t) \tilde{S}
$$

where

$$
B(t)=\left(\begin{array}{cc}
c & -1 \\
-g_{x}^{\prime}\left(t, x_{0}(t)\right) & 0
\end{array}\right),
$$

and $M(0)=I$. The Poincaré mapping of (3.5) can be expressed as

$$
P X-X_{0}=P X-P X_{0}=P\left(X-X_{0}\right)=M(2 \pi)\left(X-X_{0}\right)+o\left(X-X_{0}\right),
$$

by the differentiability of $S(t)$ with respect to the initial value $X$. It follows from $G\left(t, x_{0}(t)\right) \in \mathcal{Q}(p, n)$ and Lemma 2.5 that $M(2 \pi)$ has a pair of complex conjugate eigenvalues $\mu$ and $\bar{\mu}$ with $|\mu|=|\bar{\mu}|=e^{-c \pi}$. Hence, $M(2 \pi)$ is a contracting mapping from $\mathbb{R}^{2}$ to $\mathbb{R}^{2}$. According to Lemma 3.2 , there exists a $C^{1}$ diffeomorphism $\phi$ which is near enough to the identity mapping such that $P X-X_{0}=\left[\phi^{-1} M(2 \pi) \phi\right]\left(X-X_{0}\right)$. From elementary linear algebra, there exists an invertible constant matrix $C$ such that

$$
M(2 \pi)=C^{-1}\left(\begin{array}{cc}
\mu & 0 \\
0 & \bar{\mu}
\end{array}\right) C=C^{-1} D(\mu) C .
$$

Let $\psi=C \phi$, then

$$
P X-X_{0}=\left[\psi^{-1} D(\mu) \psi\right]\left(X-X_{0}\right) .
$$

Hence, one has

$$
\begin{aligned}
P^{2} X-X_{0} & =\left[\psi^{-1} D(\mu) \psi\right]\left(P X-X_{0}\right) \\
& =\left[\psi^{-1} D(\mu) \psi\right]\left(\left[\psi^{-1} D(\mu) \psi\right]\left(X-X_{0}\right)\right) \\
& =\left[\psi^{-1} D^{2}(\mu) \psi\right]\left(X-X_{0}\right)
\end{aligned}
$$


and inductively

$$
\begin{aligned}
P^{n} X-X_{0} & =\left[\psi^{-1} D(\mu) \psi\right]\left(P^{n-1} X-X_{0}\right) \\
& =\left[\psi^{-1} D^{n}(\mu) \psi\right]\left(X-X_{0}\right) .
\end{aligned}
$$

Note that $C$ is constant matrix, $\phi$ is near the identity. Then there exist $K_{1}, K_{2}>0$ such that if $X-X_{0}$ is small,

$$
K_{1}\left|X-X_{0}\right|<\left|\psi(X)-\psi\left(X_{0}\right)\right|<K_{2}\left|X-X_{0}\right| .
$$

Therefore, the Lyapunov exponent is given by

$$
\lim _{n \rightarrow \infty} \frac{1}{2 \pi n} \ln \left|P^{n} X-X_{0}\right|=\lim _{n \rightarrow \infty} \frac{1}{2 \pi n} \ln \left|\left[\psi^{-1} D^{n}(\mu) \psi\right]\left(X-X_{0}\right)\right|=-\frac{c}{2} .
$$

Consequently, every solution of (1.1) other than the unique $2 \pi$-periodic solution $x_{0}(t)$ decays to $x_{0}(t)$ at the rate of exponential decay $c / 2$. And the rate $c / 2$ is sharp and independently of the initial value. The proof of Theorem 1.1 is completed.

\section{Acknowledgements}

We are very grateful to the referees for their valuable suggestions, which have significantly improved a former version of this paper. We would like to thank Professor Meirong Zhang for very helpful comments.

\section{References}

[1] Alonso, J.M., Ortega, R.: Boundedness and global asymptotic stability of forced oscillator. Nonlinear Anal. 25, 297-309 (1995)

[2] Cabada, A., Cid, Á.J.: On comparison principles for the periodic Hill's equation. J. Lond. Math. Soc. 86(2), 272-290 (2010)

[3] Čepička, A., Drábek, P., Jenšiková, J.: On the stability of periodic solutions of the damped pendulum equation. J. Math. Anal. Appl. 209, 712-723 (1997)

[4] Chen, H., Li, Y., Hou, X.: Exact multiplicity for periodic solutions of Duffing type. Nonlinear Anal. 55, 115-124 (2003)

[5] Chen, H., Li, Y.: Rate of decay of stable periodic solutions of Duffing equations. J. Differ. Equ. 236, 493-503 (2007)

[6] Chen, H., Li, Y.: Existence, uniqueness and stability of periodic solutions of an equation of Duffing type. Discrete Contin. Dyn. Syst. 18, 793-807 (2007)

[7] Chicone, C.C.: Ordinary Differential Equations with Applications. Springer, New York (1999)

[8] Conway, J.B.: A course in Functional Analysis. Graduate Texts in Mathematics, 2nd edn, vol. 96. pp. 134-135. Springer, New York (1997) 
[9] Fabry, C., Mawhin, J., Nkashama, M.N.: A multiplicity result for periodic solutions of forced nonlinear second order differential equations. Bull. Lond. Math. Soc. 18, 173-180 (1986)

[10] Feng, H., Zhang, M.: Optimal estimates on rotation number of almost periodic systems. Z. Angew. Math. Phys. 57, 183-204 (2006)

[11] Johnson, R., Moser, J.: The rotation number for almost periodic potentials. Commun. Math. Phys. 84, 403-438 (Erratum) (1982)

[12] Katriel, G.: Uniqueness of periodic solutions for asymptotically linear Duffing equations with strong forcing. Topol. Methods Nonlinear Anal. 12, 263274 (1998)

[13] Korman, P., Ouyang, T.: Exact multiplicity results for two classes of periodic equations. J. Math. Anal. Appl. 194, 763-779 (1995)

[14] Lazer, A.C., McKenna, P.J.: On the existence of stable periodic solutions of differential equations of Duffing type. Proc. Am. Math. Soc. 110, 274-293 (1990)

[15] Lazer, A.C., McKenna, P.J.: Large-amplitude periodic oscillations in suspension bridges: some new connections with nonlinear analysis. SIAM Rev. 32, 537578 (1900)

[16] Liang, S.: Exact multiplicity and stability of periodic solutions for a Duffing equation. Mediterr. J. Math. 10, 189-199 (2013)

[17] Liang, S.: Existence and stability of periodic solutions of Duffing equations and equations with singularity. Doctoral Dissertation, Tsinghua University, Beijing (2010). (in Chinese)

[18] Liu, W., Li, Y.: Existence of $2 \pi$-periodic solutions for the non-dissipative Duffing equation under asymptotic behaviors of potential function. Z. Angew. Math. Phys. 57, 1-11 (2006)

[19] Llibre, J., Roberto, L.A.: On the periodic solutions of a class of Duffing differential equations. Discrete Contin. Dyn. Syst. 33, 277-282 (2013)

[20] Loud, W.S.: Periodic solutions of $x^{\prime \prime}+c x^{\prime}+g(x)=\epsilon f(t)$. Mem. Am. Math. Soc. 31, 58 (1959)

[21] Magnus, W., Winkler, S.: Hill's Equation, Dover, Now York (1966)

[22] Mawhin, J.: Global results for the forced pendulum equation. In: Cañada, A., Drábek, P., Fonda, A: Handbook of Differential Equation: Ordinary Differential Equation, vol. 1, pp. 533-589. Elsevier B.V, Amsterdam (2008)

[23] Mawhin, J.: Topological degree and boundary value problems for nonlinear differential equations. In: Furi, M., Zecca, P. (eds.) Topological Methods for Ordinary Differential Equations, Lectures Given at the first C.I.M.E. Session held in Montecatini Terme, 1991, Lectures Notes in Mathematics, vol 1537, pp. 74-142. Springer, Berlin (1993) 
[24] Njoku, F.I., Omari, P.: Stability properties of periodic solutions of a Duffing equation in the presence of lower and upper solutions. Appl. Math. Comp. 135, 471-490 (2003)

[25] Ortega, R.: Prevalence of non-degenerate periodic solutions in the forced pendulum equation. Adv. Nonlinear Stud. 13, 219-229 (2013)

[26] Ortega, R.: Some applications of the topological degree to stability theory. In: A., Frigon, M. (eds.) Topological methods in differential equations and inclusions Granas, pp. 377-409. Kluwer, Dordrecht (1995)

[27] Ortega, R.: The first interval of stability of a periodic equation of Duffing type. Proc. Am. Math. Soc. 115, 1061-1067 (1992)

[28] Ortega, R.: Stability and index of periodic solutions of an equation of Duffing type. Boll. UMI B 3, 533-546 (1989)

[29] Tarantello, G.: On the number of solutions for the forced pendulum equation. J. Differ. Equ. 80, 79-93 (1989)

[30] Torres, P.J.: Existence and stability of periodic solution of a Duffing equation by using a new maximum principle. Mediterr. J. Math. 1, 479-486 (2004)

[31] Torres, PJ: Existence of one-signed periodic solutions of some second-order differential equations via a Krasnoselskii fixed point theorem. J. Differ. Equ. 190, 643-662 (2003)

[32] Torres, P.J., Zhang, M.: A monotone iterative scheme for a second order nonlinear equation based a generalized anti-maximum principle. Math. Nachr. 251, 101-107 (2003)

[33] Van Horssen, W.T., Pischanskyy, O.V.: On the stability properties of a damped oscillator with a periodically time-varying mass. J. Sound Vib. 330, 32573269 (2011)

[34] Wang, H., Li, Y.: Existence and uniqueness of periodic solutions for Duffing equations across many points of resonance. J. Differ. Equ. 108, 152-169 (1994)

[35] Xia, D.; Wu, Z.; Yan, S.; Shu, W.: Real Analysis and Functional Analysis II, Foundations of Modern Mathematics, vol. 17, pp. 152-153. Higher Education Press, Beijing (2009). (in Chinese)

[36] Zhang, M.: Optimal conditions for maximum and antimaximum principles of the periodic solution problem. Bound. Value Probl. 2010, 1-26 (2010)

[37] Zhang, M.: Certain classes of potentials for $P$-Laplacian to be nondegenerate. Math. Nachr. 278, 1823-1836 (2005)

[38] Zhang, M.: The rotation number approach to eigenvalue of the one-dimensional p-Laplacian with periodic potentials. J. Lond. Math. Soc. 64(2), 125-143 (2001)

[39] Zhang, M., Li, W.: A Lyapunov-type stability criterion using $L^{\alpha}$ norms. Proc. Am. Math. Soc. 130, 3325-3333 (2002) 
[40] Zitan, A., Ortega, R.: Existence of asymptotically stable periodic solutions of a forced equation of Liénard type. Nonlinear Anal. 22, 993-1003 (1994)

Shuqing Liang

School of Mathematics

Jilin University

Changchun 130012

People's Republic of China

e-mail: liangshuqing@jlu.edu.cn; liangsq1982@gmail.com

Received: 23 February 2015.

Accepted: 12 October 2015. 